\title{
Bilateral carotid septic thrombosis secondary to invasive mucormycosis presented as a retropharyngeal abscess
}

Sami el Assar de la Fuente ${ }^{1}$, Julio Fernández Mata ${ }^{2 *}$, Mar Jiménez de la Peña ${ }^{2}$, Chawar Hayoun ${ }^{2}$, Ana Álvarez Vázquez ${ }^{2}$ and Youck Jen Siu Navarro $^{3}$

${ }^{1}$ Hospital Universitario Doctor Negrin, Las Palmas de Gran Canaria, Spain

${ }^{2}$ Hospital Universitario Quiron Salud Madrid, Pozuelo de Alarcón, Spain

${ }^{3}$ General Surgery Department in Hahnemann University Hospital, Philadelphia, USA

\begin{abstract}
Mucormycosis is a rapid, progressive and lethal form of fungal infections, particularly affecting immunocompromised and poorly controlled diabetic patients. It usually starts in the nose and paranasal sinuses and onrushes along the arteries and through their walls causing thrombosis and subsequent necrosis of implicated tissues.

The purpose of this article is to describe a rare case of a Mucormycosis retropharyngeal abscess in a 45-year-old diabetic type I patient that was complicated with bilateral carotid artery septic thrombosis. The patient was treated with intravenous amphotericin B, surgical drainage, never the less there were a secondary direct affection of both carotid arteries with multiple septic emboli to the brain on imaging studies and subsequent focal neurological defects on clinical evaluation. We report the computed tomographic $(\mathrm{CT})$, and magnetic resonance (MR) imaging of, what is to the best of our knowledge, the first case of a bilateral septic carotid artery thrombosis secondary to angioinvasive mucormycosis manifested as a retropharyngeal abscess.
\end{abstract}

\section{Case report}

A 45-year-old woman, Insulin dependent Diabetes mellitus, was referred for worsening of her general condition, drowsiness and difficulties in language expression in the setting of a recent history of upper respiratory tract infection for the last 3 days that was treated with antibiotics.

Cranial computed tomography (CT) findings on admission were unremarkable, and the diagnosis of a diabetic ketoacidosis state was established. When level of consciousness was recovered 24 hour later, rhinolalia and odynophagia were detected, consultation to otolaryngologist evinced a retropharyngeal abscess and a mapping $\mathrm{CT}$ of neck and chest were subsequently ordered to mark off the dimensions of affections. Contrasted enhanced CT of the neck revealed enlarged tonsils with hyper enhancement and a large retropharyngeal abscess extending to posterior cervical and parapharyngeal spaces. The diagnosis was of an acute pharyngeal- tonsillitis complicated with a retropharyngeal abscess. No implication of the mediastinum was found on Chest CT. Tracheotomy and abscess drainage were performed, antibiotic treatment with broad-spectrum regimen was established and samples for microbiological and cytological analysis were sent.

On day 14 of admission the patient developed acute neurological deficits: motor aphasia, conjugate gaze deviation to the left and an apparent right hemiparesis. Urgent cranial CT findings yielded up a subtle cortico-subcortical hypodensity in the left anterior frontal lobe with adjacent effacement of the sulci. There was an extension of the retropharyngeal abscess to both carotid spaces with enlargement and hypodensity within cervical and petrosal segments of the two internal carotid arteries (ICAs) which suggested occlusion due to septic thrombosis (Figures 1-4).

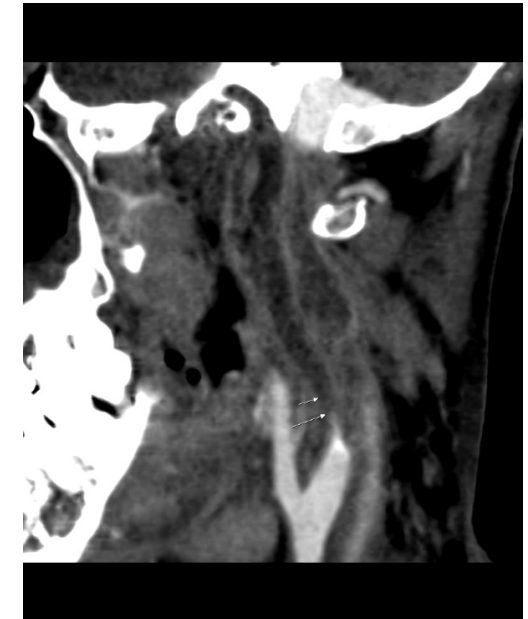

Figure 1. Sagittal contrast-enhanced CT shows a hypodense filling defect (arrows) that occupies the whole cervical segment of left internal carotid artery (ICA), compatible with total arterial occlusion in the setting of septic thrombosis

These findings of both cerebral and internal carotid arteries (ICAs) were confirmed on MRI. Diffusion weighted images (DWI) showed multiple small acute ischemic events in the territory of the two middle cerebral arteries (MCA), as well as a larger acute infarct in territory of the left anterior cerebral artery (ACA) (Figure 5). Septic thrombosis of both

${ }^{*}$ Correspondence to: Julio Fernández Mata, Radiology senior in Hospital Univeristario Quiron Salud Madrid Pozuelo de Alarcón, Spain, E-mail: julioferma@gmail.com

Received: May 10, 2018; Accepted: May 29, 2018; Published: June 01, 2018 


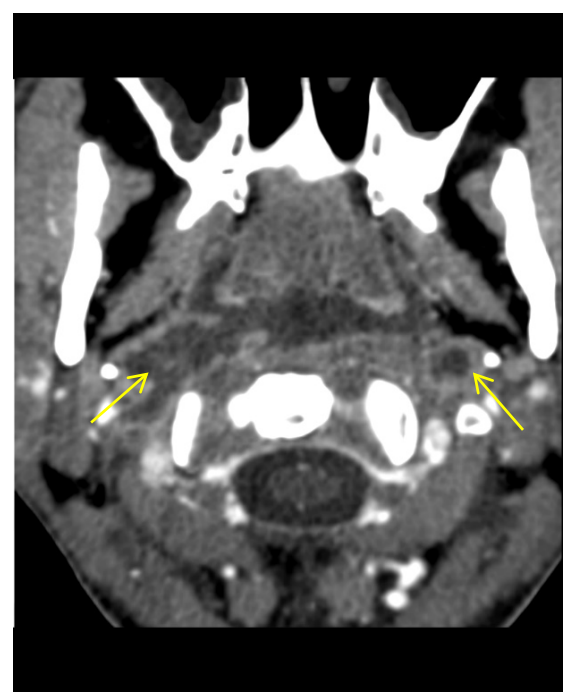

Figure 2. Axial contrast-enhanced CT scan shows invasion (arrow) of the both ICA by a retropharyngeal Mucormycosis abscess

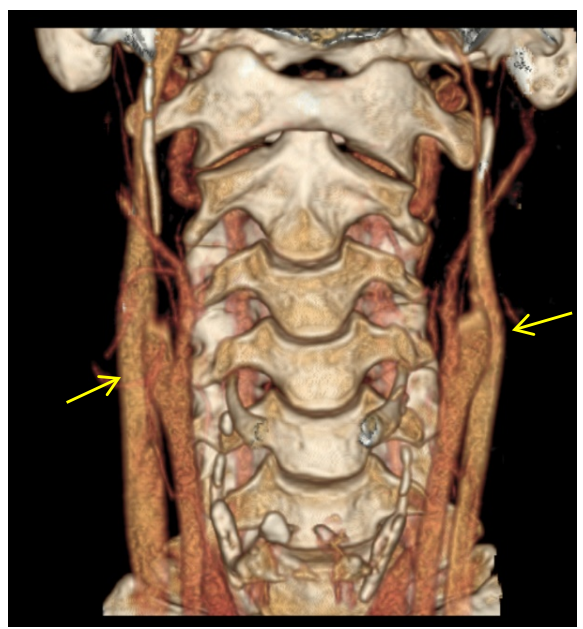

Figure 3. Surface-rendered 3D volume CT angiogram of the neck. Total occlusion of cervical segments of ICAs. Notice that internal jugular veins are spared (arrows).

ICAs was detected on Angio MRI (Figure 6). The thrombosis extended from the cervical segment up till the carotid canal. Blood flow to the anterior territory was compensated via the circle of Willis and external carotid arteries. Gadolinium administration exhibited enhancement of the carotid walls, more obvious in the initial segments of the ICAs as well as in the carotid siphons, with intact internal jugular veins. Also, there was a remarkable contrast uptake of the retropharyngeal abscess with extension to bilateral carotid and pharyngeal spaces. No variants in the circle of Willis were detected.

Comparative MRI study one week later, showed no significant changes except for the existence of a new small peripheral cortical infarction in the territory of the left posterior cerebral artery (PCA) (Figure 7). This raised attention to a possible initial failure of Willis circle's posterior territory in meeting anterior territory and brain needs in the setting of bilateral thrombosed ICAs. Finally, microbiological study yielded up Mucormycosis and the diagnosis of angioinvasive retropharingueal mucormycosis, was established.

Almost three weeks after the initial MRI, a new acute lesion was detected on DW sequences, affecting the superior aspect of the head and the anterior segment of the corpus of the caudate nucleus on the left side (Figure 8). Occlusion of almost all the trajectory of both internal carotid arteries persisted, with higher affection in cervical and petrosal segments. There was a stronger enhancement of the wall of the cervical segment on the right side, while the wall of the cervical segment of left ICA was not clearly identified (Figures 9-11).

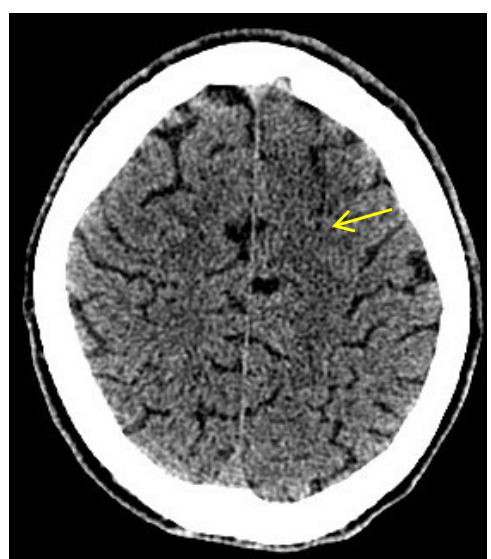

Figure 4. Axial cranial CT shows subtle cortico-subcortical hypodensity in the left anterior frontal lobe with adjacent effacement of the sulci

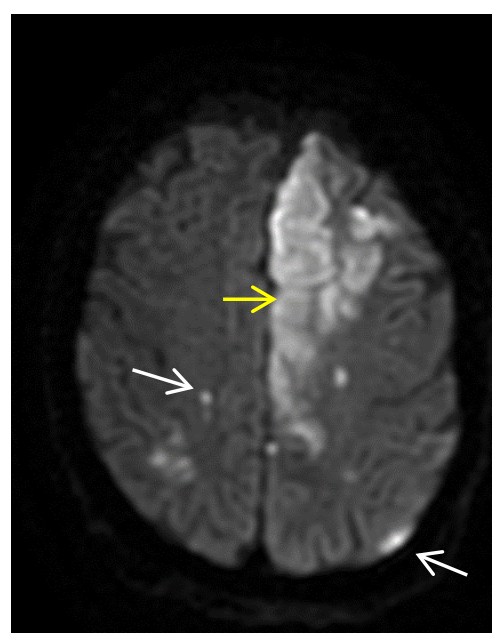

Figure 5. Axial diffusion-weighted (DW) MRI shows multiple small, acute, ischemic events in the territory of the two MCAs (white arrows). A fairly larger acute infarct is seen in territory of the left anterior cerebral artery (yellow arrow).

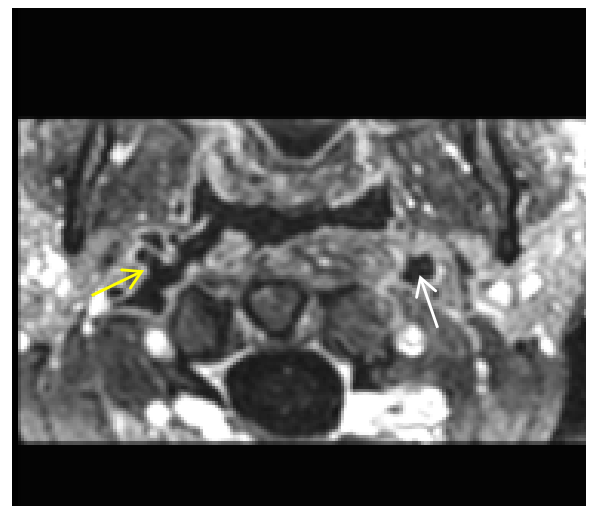

Figure 6. Contrast-enhanced (C+), fat-saturated (FatSat), T1-weighted (T1W) axial imaging stands for a better characterization of the invasion into the right ICA (yellow arrow). Notice avid enhancement of arteries' walls with hypodense lumen, indicating complete occlusion in the setting of septic thrombosis. (White arrow points to left thrombosed ICA). 


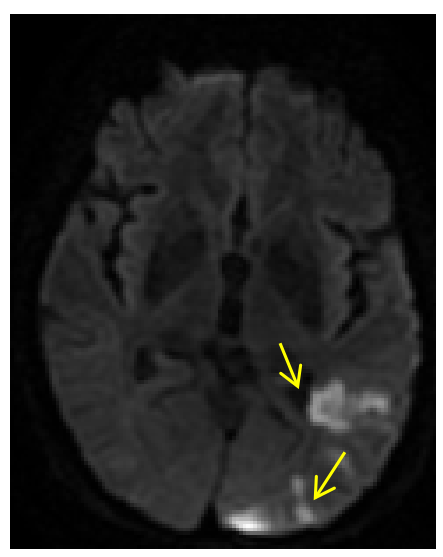

Figure 7. Axial DW MRI a week later. The restriction in diffusion in the left occipital lobe (arrow) indicates an acute ischemic event in left PCA. In the setting of occlusion of anterior territory vascularity and absence of circulation variants, this could indicate an initial failure of posterior territory in meeting anterior cerebral territory needs

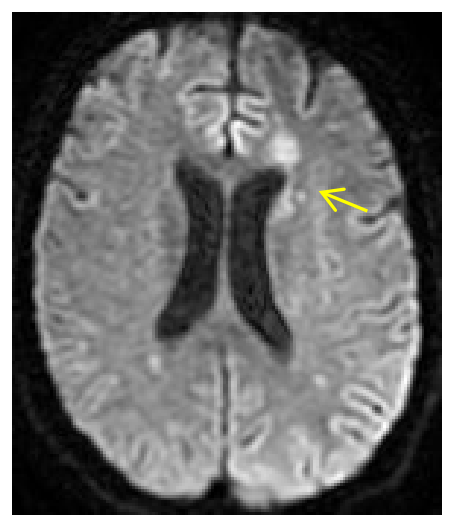

Figure 8. Axial DW MRI shows a new isquemic lesion on the superior aspect of the head and the anterior segment of the corpus of the caudate nucleus on the left side
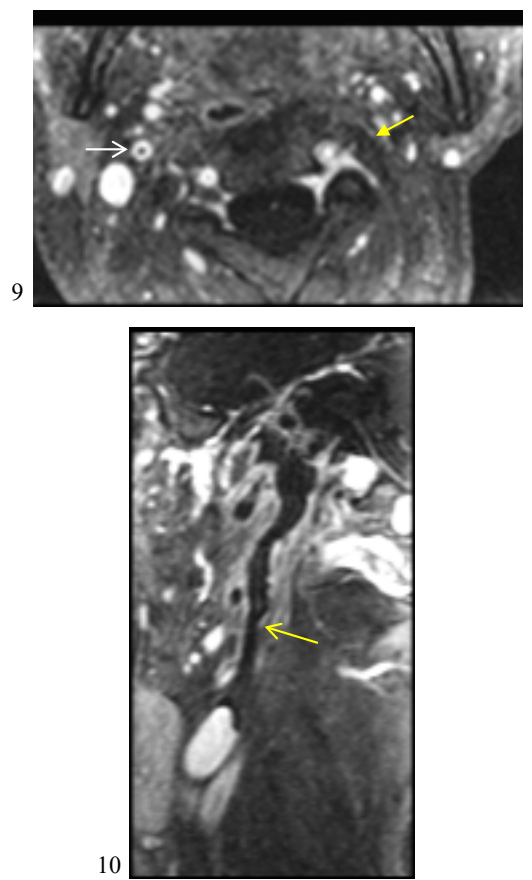

Figure 9-10. Axial, Sagittal and C+FatSat-T1W MRI has better resolution than $\mathrm{C}+\mathrm{CT}$ images. Complete thrombosis of the left ICA. Artery wall in some segments is not identified (yellow arrow). White arrow shows avid enhancement of right ICA wall

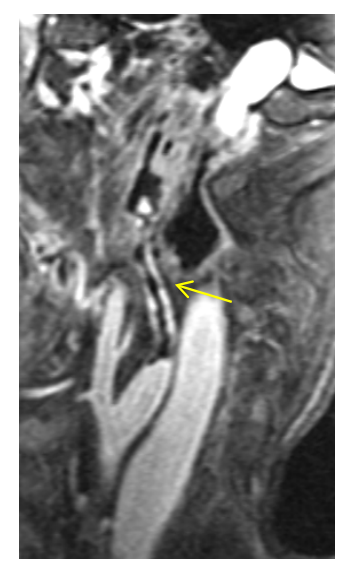

Figure 11. Sagittal C+ FatSat - T1W MRI. The right ICA shows nodularity and hyper enhancement of its wall due to invasion by Mucormycosis

The patient is still admitted to intensive care unit (ICU) with a poor prognosis.

\section{Discussion}

Mucormycosis, is a primarily opportunistic infection and member of the order Mucorales [1], and it was first described by Paulltauf A in 1885 [2]. This rapid, progressive and lethal fungi represent the third leading cause of invasive fungal infections following Aspergillus and Candida [1]. It usually starts in the nose and paranasal sinuses and onrushes along the arteries and through their walls with thrombosis and subsequent necrosis of hard and soft tissues [3].

Immunocompromised patients and poorly controlled diabetic are particularly susceptible, especially to the rhino-orbital-cerebral form of the disease $[4,5]$. Although its role is still not clearly understood, Diabetic ketoacidosis is recognized as the commonest underlying condition in the majority of rhinocerebral mucormycosis cases [2], the latter, with a percentage ranging from $30 \%$ to $50 \%$ in a large case series, it has been considered the most common form of the disease [6,7].

Some of their genera, in particularly Rhizopus organisms, one of the most prevalent found in human infections [8], have an enzyme, ketone reductase, that allow them to thrive in high glucose acidic conditions. Serum from healthy individuals inhibits growth of Rhizopus, whereas serum from individuals in diabetic ketoacidosis stimulates growth [9].

Mucormycosis is characterized by infarction and necrosis of host tissues that results from invasion of the vasculature by hyphae [10]. The infection usually presents as acute sinusitis with fever, congestion with purulent nasal discharge, headache, and sinus pain. Mucormycosis involves all the sinuses, and spread rapidly to contiguous structures, such as the palate, orbit, and brain. However, rhinoorbitocerebral mucormycosis may have an indolent course [11].

A black eschar, which only appears in $20-40 \%$ of the patients, is considered a bad prognosis sign [12]. It results from necrosis of tissues after vascular invasion by the fungus. Necrosis of the palate, destruction of the turbinates, perinasal swelling, erythema and cyanosis of the facial skin over involved sinuses are the contradistinction of spread beyond the sinuses [13]. Periorbital edema, proptosis, and blindness indicate orbital participation [14].

Clinical and radiological aspects are like those observed in other invasive filamentous fungi infections such as invasive aspergillosis, 
fusariosis or scedosporiosis [15] being cavernous sinus thrombosis one of the major complications by direct spread from neighboring sphenoid sinuses.

The Central Nervous System injury is likely due to the thrombotic occlusion of the affected blood vessels directly invaded by this aggressive mycotic infection or as a consequence of internal carotid artery thrombosis $[12,16]$.

Mainstays of treatment involve stopping immediately immunosuppressor therapy as well as corticosteroids, correction of the acidosis status, instauration of amphotericin B or other antifungals such as Posaconazole. Whenever possible, extended resection is required until bleeding tissue is found. Repeated surgery and surgical reconstruction are also considered [12,17]. The fungistatic and angiogenic effects of hyperbaric oxygen ( $\mathrm{HBO}$ ) have been described. It could improve the vascularization and enhance the effect of amphotericin B [18]. Its benefit has not been established [19].

Overall mortality from rhinoorbitalcerebral mucormycosis ranges from 25 to $62 \%$, with the best prognosis in patients with infection confined to the sinuses [8]. Functional and esthetic consequences are to be expected in the rhinocerebral forms [12].

This paper, to the best of our knowledge, is the first case reported in the literature of a bilateral septic carotid artery thrombosis secondary to angioinvasive Mucormycosis manifested as a retropharyngeal abscess. The peculiarity of the paper is owing to the combination of the unusual presentation of Mucormycosis as a retropharyngeal abscess, the direct invasion of both carotid arteries in unusual segment (away from the cavernous sinus), the adherence of the fungus to the internal elastic lamina of blood vessels which allows to spread infection along both carotid arteries leading to septic thrombosis and subsequent cerebral infarctions. This paper also, reinforces the importance of vascular studies with CT and MRI to demonstrate the invasive characteristics of this fungus, determining the extent and complications of the disease, where in our case, there is an involvement of retropharyngeal and parapharyngeal spaces, septic occlusion of carotid arteries and secondary infarction spotlighting MRI as the imaging study of choice.

Publication was decided on, because of the rarity of this entity, intending to widen the reader's scope and knowledge about this manysided disease and the important role of imaging studies along with clinical history and examinations, in setting of a high suspicion index for the diagnosis which should be made as early as possible due to the elevated mortality rate.

\section{References}

1. Pagano L, Offidani M, Fianchi L, Nosari A, Candoni A, et al. (2004) Mucormycosis in hematologic patients. Haematologica 89: 207-214. [Crossref]

2. Spellberg b, Edwards J, Ibrahim A (2005) Novel perspectives on mucormycosis: pathophysiology, presentation, and management. Clin Microbiol Rev 18: 556-569. [Crossref]

3. Nirmala SV, Lalitha V, Sivakumar N, Kumar KK, Srikanth M, et al. (2011) Mucormycosis associated with juvenile diabetes. J Indian Soc Pedod Prev Dent 29: S87-91. [Crossref]

4. Kauffman CA, Malani AN (2007) Zygomycosis: an emerging fungal infection with new options for management. Curr Infect Dis Rep 9: 435-440. [Crossref]

5. Wilson WB, Grotta JC, Schold C, Fisher LE (1979) Cerebral mucormycosis: an unusual case. Arch Neurol 36: 725-726. [Crossref]

6. McNulty JS (1982) Rhinocerebral mucormycosis: predisposing factors. Laryngoscope 92: 1140-1143. [Crossref]

7. Nithyanandam S, Jacob MS, Battu RR, Thomas RK, Correa MA, et al. (2003) Rhinoorbito-cerebral mucormycosis. A retrospective analysis of clinical features and treatment outcomes. Indian J Ophthalmol 51: 231-236. [Crossref]

8. Roden MM, Zaoutis TE, Buchanan WL, Knudsen TA, Sarkisova TA, et al. (2005) Epidemiology and outcome of zygomycosis: a review of 929 reported cases. Clin Infect Dis 41: 634-653. [Crossref]

9. Gale GR, Welch AM (1961) Studies of opportunistic fungi. I. Inhibition of Rhizopus oryzae by human serum. Am J Med Sci 241: 604-612. [Crossref]

10. Petrikkos G, Skiada A, Lortholary O, Roilides E, Walsh TJ, et al. (2012) Epidemiology and clinical manifestations of mucormycosis. Clin Infect Dis 54: S23-34. [Crossref]

11. Harril WC, Skiada A, Lortholary O, Roilides E, Walsh TJ, et al. (1996) Chronic rhinocerebral mucormycosis. Laryngoscope 106: 1292-1297.

12. Martin-Moro JG, Calleja JM, García MB, Carretero JL, Rodríguez JG (2008) Rhinoorbitocerebral mucormycosis: a case report and literature review. Med Oral Patol Oral Cir Bucal 13: E792-795. [Crossref]

13. Rajagopalan S (2005) Serious infections in elderly patients with diabetes mellitus. Clin Infect Dis 40: 990-996. [Crossref]

14. Hayat M, Mushtaq S, Saba S, Saif R (2011) Rhino-orbital-mucormycosis as presenting manifestation of gestational diabetes mellitus. Indian J Endocrinol Metab 15: S65-66. [Crossref]

15. Herbrecht R, Sabou M, Ledoux MP (2013) Clinical and radiological aspects of mucormycosis. Med Sci (Paris) 1: 19-24. [Crossref]

16. Munoz JA, Hughes A, Guo Y (2013) Mucormycosis-associated intracranial hemorrhage. Blood Coagul Fibrinolysis 24: 100-101. [Crossref]

17. McCarthy M, Rosengart A, Schuetz AN, Kontoyiannis DP, Walsh TJ (2014) Mold infections of the central nervous system. N Engl J Med 371: 150-160. [Crossref]

18. Bhutani S, Vishwanath G (2012) Hyperbaric oxygen and wound healing. Indian J Plast Surg 45: 316-24. [Crossref]

19. Ferguson BJ, Mitchell TG, Moon R, Camporesi EM, Farmer J (1988) Adjunctive hyperbaric oxygen for treatment of rhinocerebral mucormycosis. Rev Infect Dis 10: 551-559. [Crossref]

Copyright: (C)2018 de la Fuente SA. This is an open-access article distributed under the terms of the Creative Commons Attribution License, which permits unrestricted use, distribution, and reproduction in any medium, provided the original author and source are credited. 